\title{
2D Direction of Arrival Estimation using Channel State Information for UCA
}

\author{
Daulet Alibi ${ }^{1, \mathrm{a}}$, Di He, ${ }^{2, \mathrm{~b}}$, and Peilin $\mathrm{Liu}^{3, \mathrm{c}}$ \\ ${ }^{1}$ Shanghai Key Laboratory of Navigation and Location-based Services, Shanghai Jiao Tong \\ University, Shanghai, P.R. China \\ ${ }^{2}$ Shanghai Key Laboratory of Navigation and Location-based Services, Shanghai Jiao Tong \\ University, Shanghai, P.R. China \\ ${ }^{3}$ Shanghai Key Laboratory of Navigation and Location-based Services, Shanghai Jiao Tong \\ University, Shanghai, P.R. China \\ adaulet@sjtu.edu.cn, bdihe@sjtu.edu.cn, cliupeilin@sjtu.edu.cn
}

Keywords: Direction of Arrival (DOA), Uniform Circular Array (UCA), Channel State Information (CSI), Time-of-Flight (ToF), Orthogonal Frequency Division Multiplexing (OFDM)

\begin{abstract}
This paper presents technique of two dimensional direction of arrival (DOA) estimation of a source using uniform circular array (UCA) and channel state information (CSI). In orthogonal frequency division multiplexing (OFDM) system signal is simultaneously transmitted over subcarriers with different frequencies. CSI is a value that describes complex gain/attenuation and phase shift introduced by wireless channel/environment on every subcarrier. Noting the fact that CSI values among antennas and subcarriers include phase shift due to DOA and Time-of-Flight (ToF), a novel technique of virtually extending the number of antennas by estimating the DOA and ToF at the same time is described. Simulation results show that accurate DOA estimation can be achieved even with small number of antennas.
\end{abstract}

\section{Introduction}

Direction of arrival estimation (DOA) problem arouse in the middle of last century in radar and sonar systems, which are classical applications of sensor array signal processing. The main task of DOA estimation is to extract azimuth and elevation angle informations from the received signal field. Wireless technologies developed very fast during the last few decades, and nowadays millions of people are using wireless equipment in daily life. To meet the increasing requirements of data traffic more complex modulation techniques and smart antenna structures were developed, and it became possible to use array signal processing techniques in wireless communication for mobile/wireless user localization. Multiple Input Multiple Output (MIMO) is a smart antenna technology that is already used in many communication systems. ArrayTrack[1] is an indoor localization system that exploits MIMO in Wi-Fi to track users at a very fine granularity in real time indoors. In ArrayTrack Access Points (AP) overhear the transmission and compute DOA based on the received frame. But, it doesn't exploit multiple carrier property of orthogonal frequency division multiplexing (OFDM), which is implemented in widely used 802.11 W-Fi and Long-Term Evolution (LTE) standards. In [2], a novel approach called FILA explores frequency diversity of the subcarriers and leverages channel state information (CSI) values to build a propagation model for positioning with fingerprinting, while in [3] a novel location signature CSI-MIMO incorporates CSI and MIMO of each subcarrier for fingerprinting. In [4] system called SpotFi utilizes CSI information reported by commodity Wi-Fi card to simultaneously estimate DOA and Time-of-Flight (ToF) of different sources. But all these methods provide only one dimensional angle information, while nowadays DOA estimation in 2D, i.e. azimuth and elevation angles, is expected due to accuracy requirements.

This paper describes method of two dimensional DOA estimation using uniform circular arrays. In OFDM system data is sent on multiple carriers simultaneously, and channel state information value 
that represent complex attenuation and phase shift of different subcarriers. CSI among antennas include phase shift due to angle of incoming plane wave, so it is possible to extract azimuth and elevation angle informations. And CSI among different subcarriers include phase shift due to ToF, so by simultaneously extracting angles and ToF informations from CSI it is possible to virtually extend the number of antennas in UCA.

\section{System Model and Problem Formulation}

It is assumed that the array is circular with $N$ antennas, and $L$ narrowband source signals are impinging on it from the far-field. The geometry of the array is shown in Fig. 1. All array elements are assumed to be identical, omnidirectional, and uniformly distributed over the circumference of a circle of radius $R$ in the $x y$ plane. A spherical coordinate system is used to represent azimuth and elevation angles of incoming signal waves, with the origin being located at the center of the array. Elevation angle is measured down from the $z$ axis, and azimuth angle is measured counterclockwise from the $x$ axis. The $n$th antenna array is displaced from the $x$ axis by an angle $\gamma_{n}=2 \pi n / N, n=0,1, \ldots, N-1$.

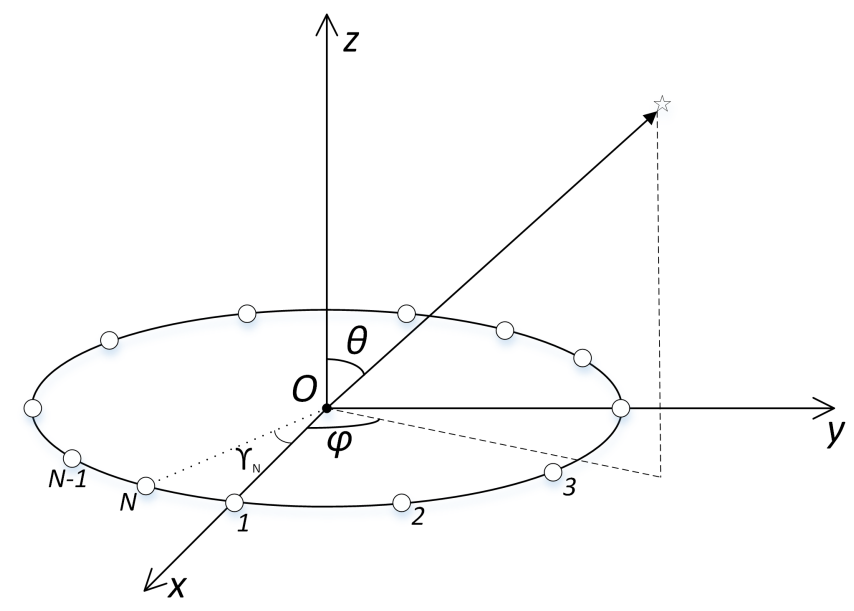

Fig. 1: UCA geometry under consideration

Due to different positions on the $x y$ plane, complex envelope of incoming signal at the origin and at $n$th element's position has phase difference $\psi_{n}=e^{j k_{0} R \sin \theta \cos \left(\varphi-\gamma_{n}\right)}[5]$, where $k_{0}=2 \pi / \lambda$ is a wavenumber of a plane wave propagating in space with speed $\lambda, \theta$ and $\varphi$ are elevation and azimuth angles respectively. The array steering vector for the $l$ th path can be written as

$$
\mathbf{a}\left(\theta_{l}, \varphi_{l}\right)=\left[\begin{array}{c}
e^{j k_{0} R \sin \theta_{l} \cos \left(\varphi_{l}\right)} \\
e^{j k_{0} R \sin \theta_{l} \cos \left(\varphi_{l}-\gamma_{1}\right)} \\
\vdots \\
e^{j k_{0} R \sin \theta_{l} \cos \left(\varphi_{l}-\gamma_{N-1}\right)}
\end{array}\right]
$$

and there are as many steering vectors as the number of sources. The output signal vector $\mathbf{x}$ of antenna array, obtained by superposition of signals from all the paths is written as

$$
\mathbf{x}=\mathbf{A s}+\mathbf{n}
$$

where $\mathbf{A}=\left[\mathbf{a}\left(\theta_{1}, \varphi_{1}\right), \ldots, \mathbf{a}\left(\theta_{L}, \varphi_{L}\right)\right]$ is an array manifold, matrix with $N$ rows and $L$ columns, $\mathbf{s}=$ $\left[\alpha_{1} e^{j 2 \pi f_{c} \tau_{1}}, \ldots, \alpha_{L} e^{j 2 \pi f_{c} \tau_{L}}\right]^{T}$ is a vector of complex envelopes received by antennas with attenuation $\alpha$ and phase shift due to ToF along $L$ paths, and $n$ is a vector of additive white Gaussian noise (AWGN). In OFDM system data is transmitted over multiple subcarriers with different frequency simultaneously. 
CSI value represents overall attenuation and phase shift introduced by the channel at each antenna and each subcarrier. Having $N$ antennas and $K$ subcarriers, CSI matrix can be written as

$$
C S I=\left[\begin{array}{cccc}
c s i_{1,1} & c s i_{1,2} & \ldots & c s i_{1, K} \\
c s i_{2,1} & c s i_{2,2} & \ldots & c s i_{2, K} \\
\vdots & & & \\
c s i_{N, 1} & c s i_{N, 2} & \ldots & c s i_{N, K}
\end{array}\right]
$$

where $c s i_{n, k}$ is the CSI value at the $n$th antenna and $k$ th subcarrier. Obviously, CSI values among different subcarriers have different phase shifts, this is because each subcarrier passes through the same path or travels with the same ToF but on different carrier frequency. For example, in OFDM system with equally spaced subcarriers, phase shift difference between two adjacent subcarriers is

$$
\Omega\left(\tau_{l}\right)=e^{-j 2 \pi f_{\delta} \tau_{l}}
$$

where $f_{\delta}$ is frequency spacing between the subcarriers. And phase shifts of CSI values among different antennas are different due to DOA of received signal. Combining Eq. 2 and Eq. 4 we can express CSI matrix as below

$$
C S I=\left[\begin{array}{cccc}
e^{j \vartheta \cos (\varphi)} \alpha & e^{j \vartheta \cos (\varphi)} \alpha \Omega_{\tau} & \cdots & e^{j \vartheta \cos (\varphi)} \alpha \Omega_{\tau}^{K-1} \\
e^{j \vartheta \cos \left(\varphi-\gamma_{1}\right)} \alpha & e^{j \vartheta \cos \left(\varphi-\gamma_{1}\right)} \alpha \Omega_{\tau} & \cdots & e^{j \vartheta \cos \left(\varphi-\gamma_{1}\right)} \alpha \Omega_{\tau}^{K-1} \\
\vdots & \vdots & \ddots & \vdots \\
e^{j \vartheta \cos \left(\varphi-\gamma_{N-1}\right)} \alpha & e^{j \vartheta \cos \left(\varphi-\gamma_{N-1}\right)} \alpha \Omega_{\tau} & \cdots & e^{j \vartheta \cos \left(\varphi-\gamma_{N-1}\right)} \alpha \Omega_{\tau}^{K-1}
\end{array}\right]
$$

where $\vartheta=k_{0} R \sin (\theta)$. In fact, received signal vector $\mathbf{x}$ described in Eq. 2 is a first column of CSI matrix in Eq. 5. But here, in the first column, phase shift introduced by ToF is equal to 1 (or in other words is absorbed into phase shift due to DOA). This assumption is made because the CSI values of first subcarrier on all antennas have reference phase shift due to ToF, and CSI values of consecutive subcarriers have $\Omega_{\tau}$ with the increasing power.

By stacking CSI values from all subcarriers and all antennas we construct modified output signal vector $\hat{\mathbf{x}}$, with steering vector having $N \times K(N$ antennas by $K$ subcarriers $)$ rows

$$
\hat{\mathbf{a}}(\theta, \varphi, \tau)=[\overbrace{\Theta \Phi_{0}, \ldots, \Theta \Phi_{0} \Omega^{K-1}}^{\text {First antenna }}, \ldots, \underbrace{\Theta \Phi_{N-1}, \ldots, \Theta \Phi_{N-1} \Omega^{K-1}}_{\text {Last antenna }}]
$$

Thus, number of antennas in the modified steering vector of an array virtually extended by the number of subcarriers. Here, steering vector has elevation dependence through $\Theta=e^{j \vartheta}$, azimuth dependence through $\Phi_{n}=e^{j \cos \left(\phi-\gamma_{n}\right)}, n=0,1, \ldots, N-1$, and ToF dependence through $\Omega$. Number of steering vectors is equal to number of sources, and modified array manifold $\hat{\mathbf{A}}$ is constructed by stacking all steering vectors columnwise. Now, modified output vector $\hat{\mathbf{x}}$ can be written as

$$
\hat{\mathbf{x}}=\hat{\mathbf{A}} \zeta+\mathbf{n}
$$

where $\boldsymbol{\zeta}=\left[\zeta_{1}, \zeta_{2}, \ldots, \zeta_{L}\right]$ is a row vector of attenuations along $L$ paths.

The main task here is to extract angle information from vector $\hat{\mathbf{x}}$. MUSIC algorithm works with this vector. The MUSIC algorithm at a basic level conducts characteristic decomposition for the covariance matrix of an array signal output (covariance matrix $\hat{\mathbf{x}} \hat{\mathbf{x}}^{H}$ of modified output vector in our case). which results in a signal and a noise subspace. Noise subspace consists of eigenvectors corresponding to eigenvalue zero. And main assumption of MUSIC algorithm is that these eigenvectors are orthogonal to the steering vectors in array manifold[6]. That is true when array manifold is tall, which means that the number of rows is larger than the number of columns, or in other words number of antennas in the array must be large enough, at least be larger than the number of distant sources. In our case, as it was 
said before, using phase shift of complex envelope included in CSI values due to ToF number of rows in array manifold is extended, thus making matrix $\hat{\mathbf{A}}$ tall. MUSIC algorithm for UCA using CSI can be summarized as follows

Algorithm summary

1. Form sample covariance matrix $\mathbf{R}=\frac{1}{P} \sum_{i=1}^{P} \hat{\mathbf{x}} \hat{\mathbf{x}}^{H}$, where $P$ is the number of OFDM packets used for estimation.

2. Perform eigenvalue decomposition of the matrix $\mathbf{R}$. Order eigenvalues of $\mathbf{R}$ to be $\lambda_{1} \geq \cdots \geq$ $\lambda_{N K}$, and corresponding eigenvectors to be $\left[\mu_{1}, \ldots, \mu_{N K}\right]$. Let eigenvectors corresponding to $L$ largest eigenvalues span signal subspace $\mathbf{S}=\left[\mathbf{s}_{1}, \ldots, \mathbf{s}_{L}\right]$, and remaining eigenvectors span noise subspace $\mathbf{G}=\left[\mu_{L+1}, \ldots, \mu_{N K}\right]$.

3. Construct spatial spectrum $P_{M U S I C}(\theta, \phi, \tau)=\frac{1}{\mathbf{a}^{H}(\theta, \varphi, \tau) \mathbf{G G}^{h} \mathbf{a}(\theta, \varphi, \tau)}$

4. Estimate DOA by searching for peaks

\section{Simulation Results}

To demonstrate performance of the described method several simulations were performed. A UCA with only 3 omnidirectional antennas and with $\frac{\lambda}{2}$ antenna spacing were employed. First, we performed simulation of DOA estimation of one source in the far field of the array with arrival angles given by $(\theta, \varphi)=\left(90^{\circ}, 51^{\circ}\right)$. We assumed to have CSI values of 3 consecutive OFDM packets received at the UCA, and that communication system used 64 subcarriers for data transmission, but CSI values of only 60 subcarriers were used for DOA estimation. Estimated DOAs are in radians. Fig. 2 shows the root mean square (RMS) DOA error as a function of SNR after 1000 trials.
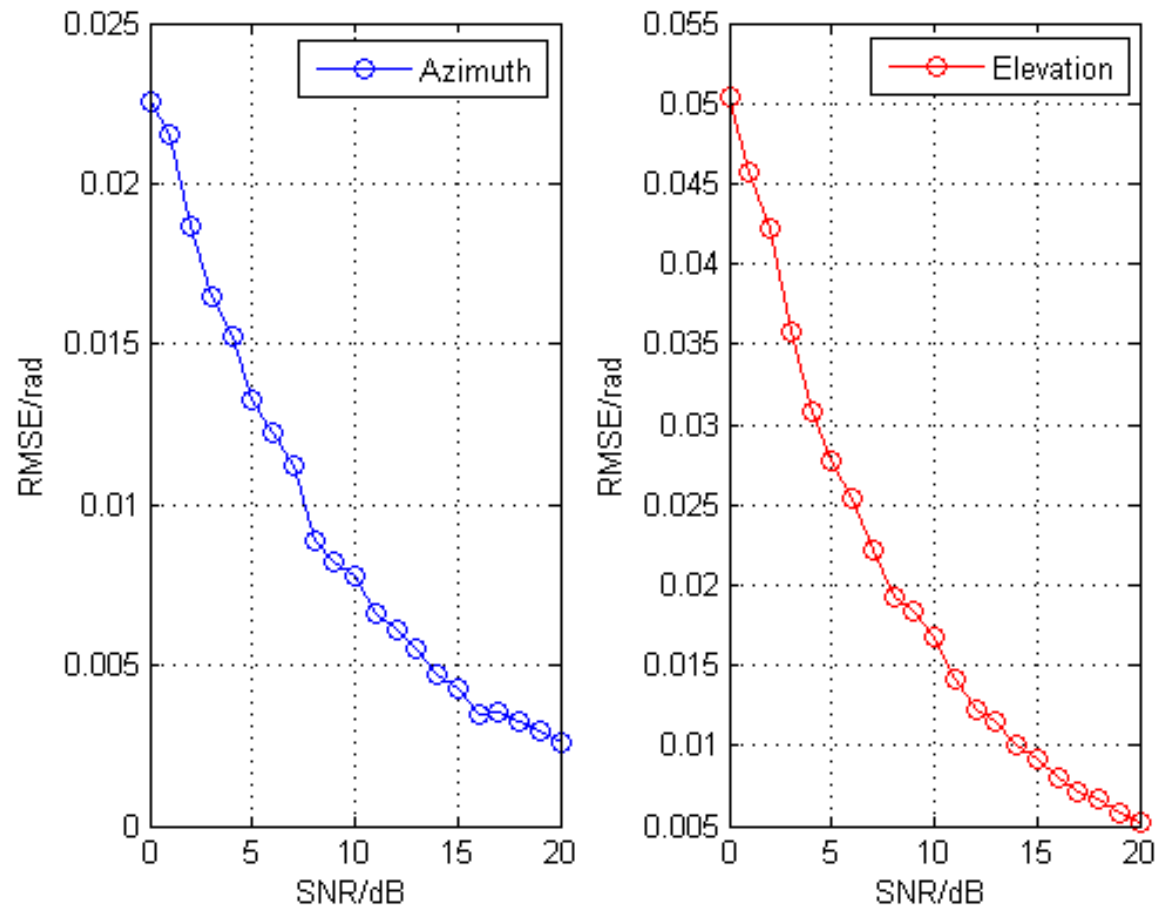

Fig. 2: Result of 1000 Monte Carlo simulations

Next, we perfromed simulations of DOA estimation of two equipowered sources in the far field of the array with $\left(\theta_{1}, \varphi_{1}\right)=\left(51^{\circ}, 90^{\circ}\right)$ and $\left(\theta_{2}, \varphi_{2}\right)=\left(72^{\circ}, 78^{\circ}\right)$. First source were assumed to have 
ToF equal to $50 \mathrm{~ns}$ and second source's ToF equal to $100 \mathrm{~ns}$. We compared described method with UCA-ESPRIT[5] using 8 antennas, 64 snapshots and same DOAs. Fig. 3 shows simulation results after 1000 trials
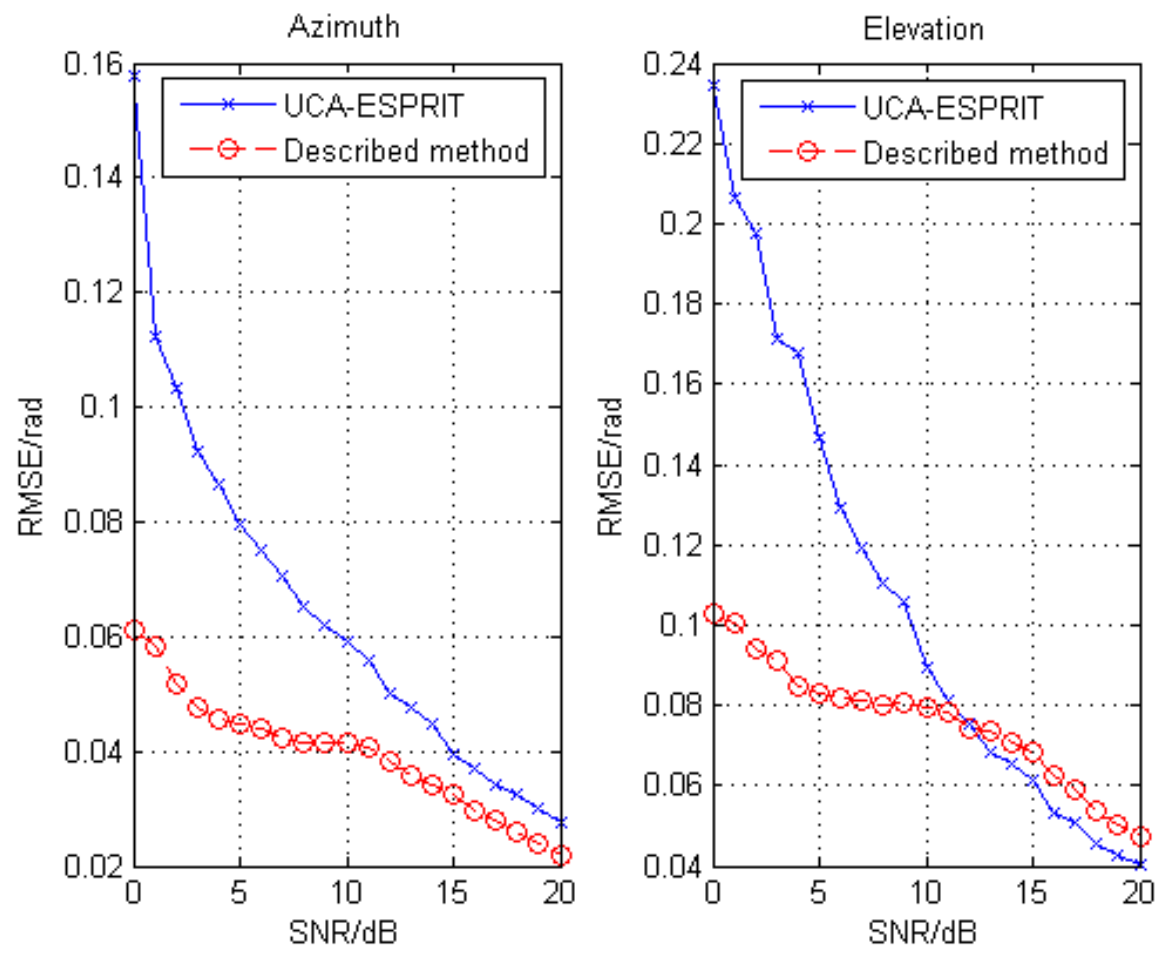

Fig. 3: Result of 1000 Monte Carlo simulations

\section{Conclusion}

A novel technique of virtually extending the number of antennas for DOA estimation process were described. DOA and ToF of far field sources were simultaneously estimated using CSI of 3 consecutive packets. Simulation results verified that the described method can achieve comparable performance even with very small number of antennas in UCA. Method of 2D DOA estimation with UCA using CSI only from one OFDM packet is leaved for future work.

\section{Acknowledgment}

This research work is supported by the Important National Science and Technology Specific Project of China under Grant No. 2016ZX03001022-006, the National Natural Science Foundation of China under Grant Nos. 91438113, 61402283, 61573242, 61401501 and 61304225, the National Science and Technology Major Project under Grant No. GFZX0301010708, and the Shanghai Science and Technology Committee under Grant Nos. 16DZ1100402 and 15511105100.

\section{References}

[1] J. Xiong and K. Jamieson, "Arraytrack: A fine-grained indoor location system," in Presented as a part of the 10th USENIX Symposium on Networked Systems Design and Implementation (NSDI 13), Lombard, IL: USENIX, 2013, pp. 71-84, [Online]. Available: https://www.usenix.org/conference/nsdi13/technical-sessions/presentation/xiong 
[2] K. Wu, J. Xiao, Y. Yi, D. Chen, X. Luo, and L. M. Ni, "Csi-based indoor localization,” IEEE Transactions on Systems, Man. and Cybernetics, Part C (Applications and Reviews), vol. 37, no. 6, pp. 1067-1080, Nov 2007.

[3] Y. Chapre, A. Ignjatovic, A. Seneviratne, and S. Jha, "Csi-mimo: Indoor location system," in 39th Annual IEEE Conference on Local Computer Networks, Sept. 2014, pp. 202-209

[4] M. Kotaru, K. R. Joshi, D. Bharadia, and S. Katti, "Spotfi: Decimeter level localization using wifi," Computer Communication Review, vol. 45., no. 5, pp 269-282, 2015. [Online]. Available: http://doi.acm.org/10.1145/2829988.2787487

[5] C. P. Mathews and M. D. Zoltowski, "Eigenstructure techniques for 2-d angle estimation with uniform circular arrays," IEEE Transactions on Signal Processing, vol. 42, no.9, pp. 2395-2407, Sep 1994.

[6] R. Schmidt, "Multiple emitter location and signal parameter estimation," IEEE Transactions on Antennas and Propagation, vol.34, no.3, pp. 276-280, Mar 1986. 